\title{
EFFECT OF ILLUMINATION IN CHROMA KEY EFFECT IN THE REALIZATION OF VIRTUAL TELEVISION STUDIO
}

\author{
Vladimir Maksimović ${ }^{1 *}$, \\ Mile Petrović ${ }^{1}$, \\ Branimir Jakšić ${ }^{1}$, \\ Ratko Ivković ${ }^{1}$, \\ Ivana Milošević ${ }^{2}$
}

\author{
${ }^{1}$ Faculty of Technical Sciences, \\ University of Priština, \\ Kosovska Mitrovica, Serbia \\ ${ }^{2}$ High School of Electrical Engineering and \\ Computing of Vocational Studies, \\ Belgrade, Serbia
}

\section{Correspondence:}

Vladimir Maksimović

e-mail:

vladimir.maksimovic@pr.ac.rs

\begin{abstract}
:
This paper analyzes the effect of front and back light on the Chroma key effect in a virtual TV studio. The model of the system in which the experiment was realized is given. Different intensity of front and back light was used. The analysis of the front light of 200 lux, 800 lux and 1400 lux was done, while the back light changes in the range from 200 to 1400 lux. The characteristics of the analyzed images were clearly determined by their luminance histogram and parameters Mean, Median, and Standard Deviation. Chroma key effect parameters: Color Tolerance, Edge Thin and Edge Feather were used for the analysis. The results that are considered are tabulated and graphically based on the influence of light on the quality of the realization of the Chroma key effect.
\end{abstract}

Keywords:

virtual studio, chroma key, color tolerance, light, luminance histogram.

\section{INTRODUCTION}

A virtual television (TV) studio allows a natural relationship between the participants and the generated scenery from the computer by realizing the correction of the generated scenery from the computer. Computer-generated scenery has the visual appearance of the real decor in the background. In this way, it is possible to create different decors, and also surreal, which creates a visual impression that the TV studio is much larger than it is [1]. The virtual studio technology is based on the Chroma key process $[2,3,4]$. It represents a way of mixing two video signals, in which the monochrome background in one video signal (live video signal from the scene) is replaced by another video signal. The replacement is done by a quick switch circuit which alternates between the foreground video signal and the background video signal or application of software packages such as Vmix, Elements or Adobe Premiere. The process of replacing the background is called keying $[5,6]$. Inserting the video signal of the new background into the monochrome background of the video signal of the foreground is done at the scanning time, at the boundary between the object or the participants in the foreground and the monochrome backgrounds. This creates the impression that the objects in the foreground are in the scene shown by a picture of another source. A monochrome background can be of any color, provided that 
this color is not on the objects or participants in the front of the scene [7]. Today, blue and green colors are used for the background [8]. An example of the realization of a Chroma key effect for a blue background is shown in Fig. 1 [9].

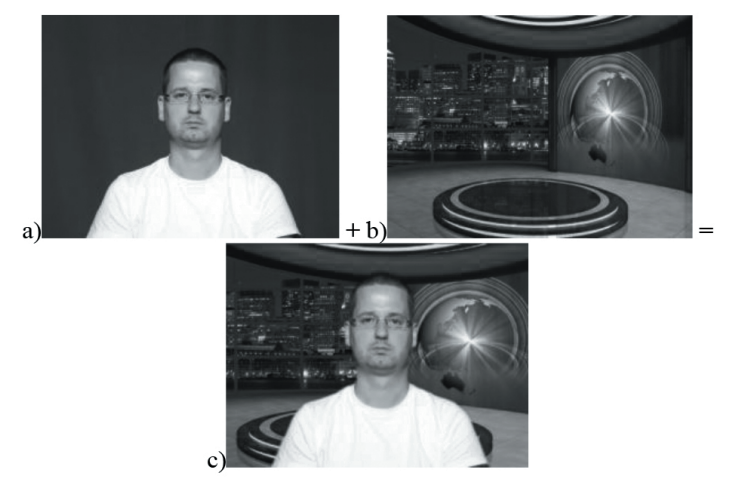

Fig. 1. Illustration of Chroma key effect: a) foregraund frame, b) background frame, c) composite frame.

For lighting is commonly used setting that includes three lights (front, side and back) [10]. The front (direct) light is set from the camera direction from $0^{\circ}$ to $30^{\circ}$, on each side, the vertical angle is about $45^{\circ}$. This setting of lights illuminate most of the face, and the smaller part remains in the shade.

Side light is set from $45^{\circ}$ to $90^{\circ}$ from either side of the camera, or $90^{\circ}$ from the main beam. It's safest to place it at $45^{\circ}$ from the camera. It should be lower than the main light.

The backlight is set against the camera from $135^{\circ}$ to $180^{\circ}$ on each side. It is placed directly behind the subject being recorded, and in relation to the camera on which large plans are made. The back light color temperature is not as critical as for the main and complementary light, so that the intensity can be reduced by dimers. Changing the color temperature to $\pm 1500 \mathrm{~K}$ is tolerated, as the human eye does not notice them. The backlight emphasizes the third dimension of the image, separates the subject from the background, and separates the foreground from the background. The background brightness intensity should be about $2 / 3$ of the main intensity of the elements on the scene. By this, it is achieved that the central object of the recording is separated from the background [11, 12].

Light sources are characterized by color temperature. The color temperature $(0 \mathrm{~K})$ is the temperature during which the black body should be heated - (light source) to make its color the colorimetrically most similar color of the medium-day light source. Illumination is the amount of light energy that falls on the unit surface in every second. The units are lux (lx) and foot-candle (fc) [13].

\section{SYSTEM MODEL}

The analysis of the influence of illumination was performed on the images obtained in the TV studio area of $12 \times 8=96 \mathrm{~m}^{2}$ and height $4.5 \mathrm{~m}$. Dimensions of the blue background are $8 \times 3.5 \mathrm{~m}$. The light distribution in relation to the object on the scene is shown in Fig. 2.

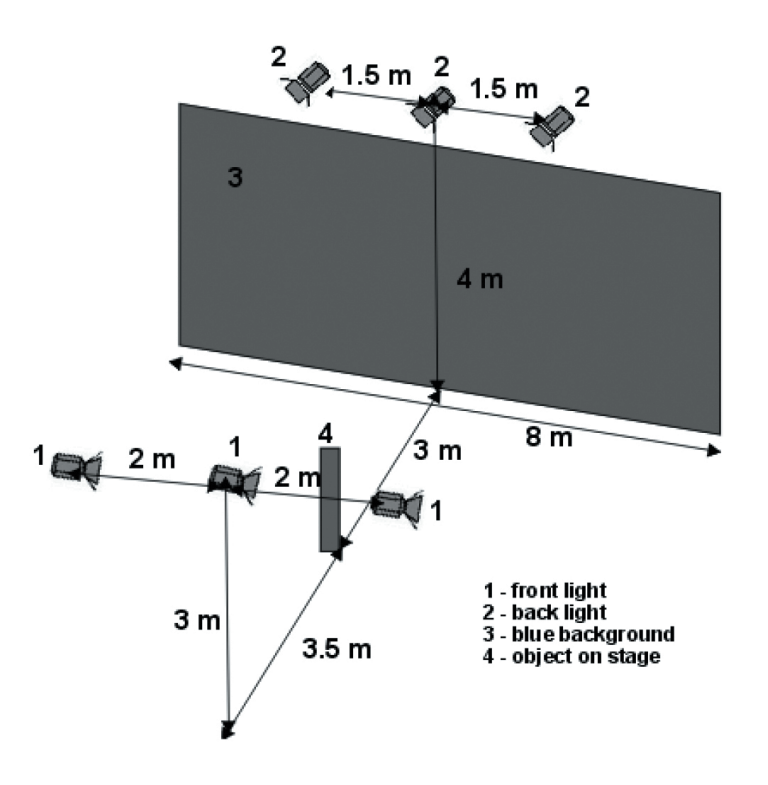

Fig. 2. Layout of lights in TV studio.

The number of front and back lights is 3 , and all of them are set at an angle of $45^{\circ}$ in relation to the horizontal plane. The horizontal distance between the front and back lights is $6.5 \mathrm{~m}$. The distance between the back lights is $1.5 \mathrm{~m}$ and is set at a height of $4 \mathrm{~m}$ in relation to the scene. Devices which are used for the formation of the back lights are Reflector Fluorescent soft lights 2 lamps $55 \mathrm{~W}, 3200 \mathrm{~K}$. The front lights are set at a height of $3 \mathrm{~m}$ in relation to the scene, and with a mutual distance of $2 \mathrm{~m}$. Devices which are used for the formation of the front lights are Reflectors with Fresnel lens (150 mm Fresnel lens and G22 lamp holder, halogen lamp $1000 \mathrm{~W}, 3200 \mathrm{~K})$.

The color temperature control instrument Minolta Color Meter II [14] was used to control the color temperature. The digital VDVM1300 digital lux meter is used to control the brightness of the light [15]. 
In this analysis, the recording subject is at a distance of $3 \mathrm{~m}$ from the background (and from the back light) and $3.5 \mathrm{~m}$ from the front light. A Canon EOS 550D digital camera [16] was used to shoot a scene on the scene, and it was set at a distance of $2 \mathrm{~m}$ from the recording subject.

In practical cases, the subject on the scene can be placed at any distance between the front and back lights.

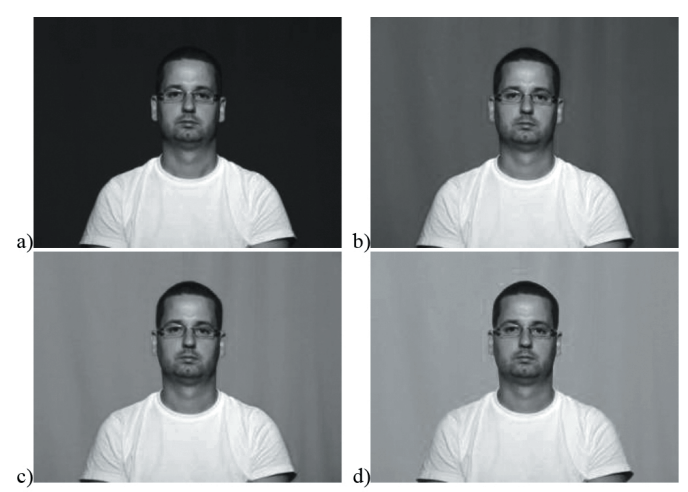

Fig. 3. Images obtained for front light 200 lux and back: a) 200 kux, b) $600 \operatorname{lux}$, c) 1000 lux, d) 1400 lux.
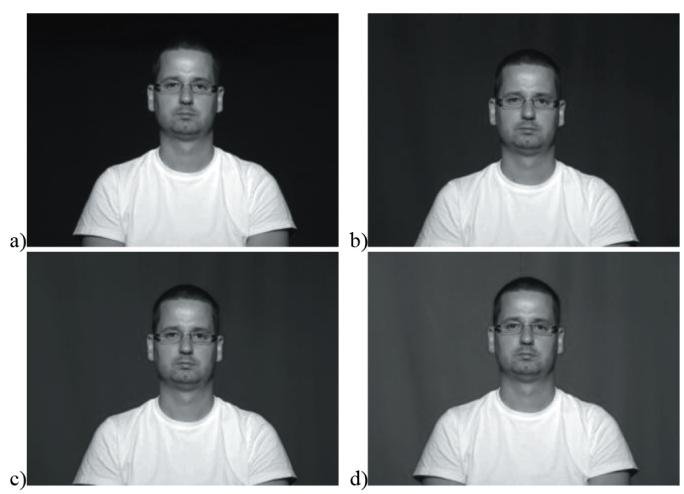

Fig. 4. Images obtained for front light 800 lux and back: a) 200 kux, b) $600 \operatorname{lux}$, c) $1000 \operatorname{lux}$, d) 1400 lux.
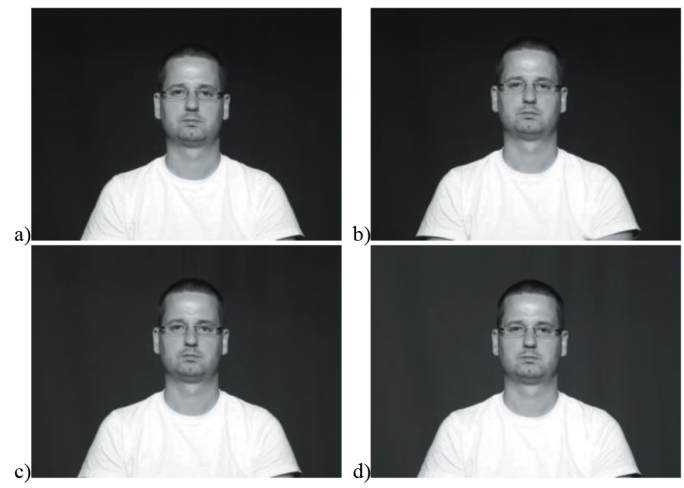

Fig. 5. Images obtained for front light 1400 lux and back: a) 200 kux, b) 600 lux, c) 1000 lux, d) 1400 lux.
For the analysis 24-bit uncompressed images were used with an original resolution of $1600 \times 1200$ pixels, realized in the previously described virtual studio. The images are illuminated by different brightness of the front and back light. Image analysis is divided into three blocks. The first one is for a fixed front light to 200 lux, the second one for fixed front light to 800 lux, and the third one for a fixed front light to 1400 lux. For all three cases, the back light changes in the range of 200 to 1400 lux. The analysis is performed on several images that are shown in Fig. 3, Fig. 4 and Fig. 5.

For the software generation of the Chroma key effect, the Adobe Premiere software package was used with the following parameters:

- Color Tolerance - Widens or reduces the color range to be transparent. More values increase the scope. It is very important because it controls the width of the color shade range that will be recognized as the corresponding key color. More values increase the angle of the arc covered by highlighted region on the color circle.

- Edge Thin - Setting the width of the border key areas (area's keyed). Positive values magnify the mask, enlarge the transparent surface, and negative values reduce the mask, or reduce the transparent surface.

- Edge Feather - Determines the edge softness. More values create soft edges.

\section{THE EFFECT OF ILLUMINATION ON THE CHANGE OF PARAMETERS COLORED BACKGROUND}

In order to make the impact of the studio light on the analyzed images the clearer luminance histograms of the analyzed images were made clearer. In Fig. 6, Fig. 7 and Fig. 8, the luminance histograms for the analyzed images with different studio light values are provided.

The luminance histogram shows the distribution of the illumination on image. The $\mathrm{X}$ axis represents hues of illumination from completely black to completely white where the spectrum divided into 256 levels, from 0 to 255. The $Y$ axis stand for representation of illumination on image. For each level, the $\mathrm{Y}$ axis shows the number of points in the image that have this level of illumination. The $\mathrm{Y}$ axis is relative because it depends on the dimensions of the image. Luminance histograms are more accurate than RGB histograms when describing perceived light distribution or "lighting" within the image. 
Luminance histograms monitor the location of each pixel in color, while RGB histograms reject this information. The RGB histogram produces three independent histograms and then connects them to one, regardless of whether each color comes from the same pixel. The light takes into account the fact that the human eye is more sensitive to the green light than red or blue light. Each pixel in the histogram of illumination represents the brightness based on the average three colors on that pixel. This average is that green represents $59 \%$ of reduced light, while red and blue channels make up only $30 \%$ and $11 \%$, respectively. Once all the pixels have been turned into light, the histogram of light is produced by counting the number of pixels on each brightness - identical to the one produced by histogram for one color [17, 18].

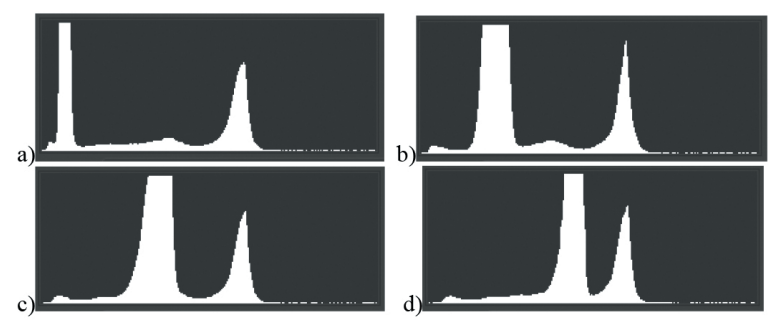

Fig. 6. The luminance histogram of the images obtained for the front light 200 lux and the back:

a) 200 lux, b) 600 lux, c) 1000 lux, d) 1400 lux.

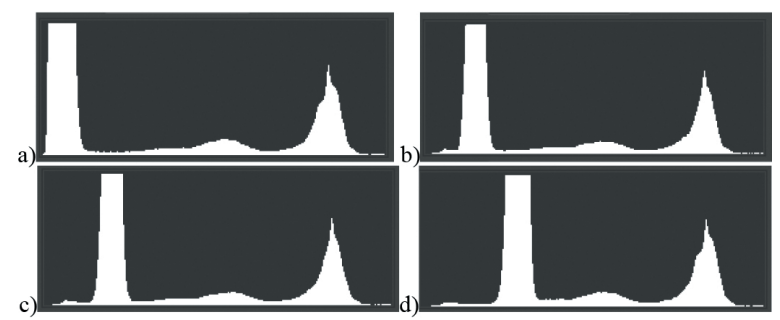

Fig. 7. The luminance histogram of the images obtained for the front light 800 lux and the back:

a) 200 kux, b) 600 lux, c) 1000 lux, d) 1400 lux.

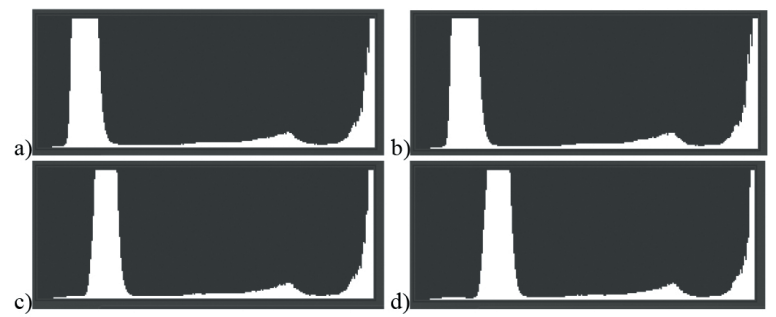

Fig. 8. The luminance histogram of the images obtained for the front light 1400 lux and the back:

a) 200 kux, b) 600 lux, c) 1000 lux, d) 1400 lux.
From the given histogram we can see that there are three different tops, one for each color. Since each image contains primarily color blue then red to the smaller extent and lastly green, the relative heights clearly indicate which color it belongs to. The relative horizontal position of each top is in accordance with the percentages used by the means of three calculation colors: $59 \%, 30 \%$ and $11 \%$. The histogram shows that the largest distribution is blue color and least red color, because the blue color is dominant in the image. However, it can also be seen that the best distribution of lighting provides an 800 lux illumination. Also, the histogram shows thet there is a loss of brighter parts of the information from image when the front light is of 200 lux, while when is the front light is of 1400 lux there is less loss of information on both sides. But still the results are much better than at 200 lux.

The characteristics of the luminance histogram are defined with three parameters: Mean, Standard Deviation and Median. Mean is the average value (brightness or brightness) of all pixels. Medain is medium brightness (brightness or light) in the distribution of tones. Standard deviation is a statistical number indicating the variation of the tone, that is, reflecting the average difference of all pixels from the mean value [19].

Table 1 gives the values of the Mean, Standard Deviation, and Median parameters obtained for the luminance histogram of the analyzed images with different intensity of the front and back illumination. From Table 1 it can be seen that Mean and Median grow with increasing back light illumination, while the range of the Median parameter decreases for higer values of front light. Standard deviation decreases with increasing illumination. Decay is significantly more pronounced for poorer front lighting. Low values of standard deviation, below 30 (which are more pronounced at lower illuminated front), usually indicate a lack of tone bandwidth, while more than 90 values (which are more pronounced at a higher illuminated front) usually indicate a very large contrast. The captured scene with a good tone range has values somewhere between 40 and 60 , so it can be seen from Table 1 that these values correspond most closely to the 800 lux front lighting. 


\begin{tabular}{|c|c|c|c|c|}
\hline $\begin{array}{c}\text { Front light } \\
\quad[\operatorname{lux}]\end{array}$ & $\begin{array}{c}\text { Back light } \\
\text { [lux] }\end{array}$ & Mean & Standard Deviation & Median \\
\hline \multirow{7}{*}{200} & 200 & 49.27 & 53.31 & 19 \\
\hline & 400 & 57.99 & 48.58 & 32 \\
\hline & 600 & 77.02 & 39.25 & 61 \\
\hline & 800 & 86.68 & 33.8 & 75 \\
\hline & 1000 & 98.67 & 29.05 & 93 \\
\hline & 1200 & 106.18 & 26.84 & 102 \\
\hline & 1400 & 115.62 & 24.22 & 115 \\
\hline \multirow{7}{*}{800} & 200 & 68.57 & 80.85 & 21 \\
\hline & 400 & 70.02 & 73.32 & 29 \\
\hline & 600 & 77.04 & 67.64 & 40 \\
\hline & 800 & 83.36 & 67.07 & 46 \\
\hline & 1000 & 86.50 & 63.57 & 52 \\
\hline & 1200 & 86.35 & 60.48 & 64 \\
\hline & 1400 & 105.37 & 56.43 & 76 \\
\hline \multirow{7}{*}{1400} & 200 & 88.09 & 85.93 & 40 \\
\hline & 400 & 87.87 & 88.38 & 38 \\
\hline & 600 & 92.55 & 87.46 & 41 \\
\hline & 800 & 95.16 & 83.83 & 47 \\
\hline & 1000 & 100.62 & 80.22 & 55 \\
\hline & 1200 & 100.91 & 80.68 & 55 \\
\hline & 1400 & 107.62 & 75.85 & 65 \\
\hline
\end{tabular}

Table 1. Luminance histogram values

\section{RESULTS AND DISCUSSION}

Table 2, Table 3 and Table 4 show the obtained values of the parameters for the realized Chroma key effect for different values of the back light, and for the front light of 200 lux, 800 lux and 1400 lux, respectively.

\begin{tabular}{ccccc}
\hline $\begin{array}{c}\text { Back } \\
\text { light } \\
{[\mathbf{l u x}]}\end{array}$ & $\begin{array}{c}\text { Color } \\
\text { Code }\end{array}$ & $\begin{array}{c}\text { Color } \\
\text { Toler- } \\
\text { ance }\end{array}$ & $\begin{array}{c}\text { Edge } \\
\text { Thin }\end{array}$ & $\begin{array}{c}\text { Edge } \\
\text { Feather }\end{array}$ \\
\hline $\mathbf{2 0 0}$ & $\# 0 \mathrm{~B} 1224$ & 18 & 2 & 7 \\
\hline $\mathbf{4 0 0}$ & $\# 11214 \mathrm{C}$ & 23 & 3 & 6 \\
\hline $\mathbf{6 0 0}$ & $\# 1 \mathrm{C} 3 \mathrm{E} 97$ & 62 & 3 & 2.5 \\
\hline $\mathbf{8 0 0}$ & $\# 2553 \mathrm{C} 2$ & 81 & 3 & 1 \\
\hline $\mathbf{1 0 0 0}$ & $\# 2963 \mathrm{E} 1$ & 116 & 4 & 0.5 \\
\hline $\mathbf{1 2 0 0}$ & $\# 2 \mathrm{~A} 6 \mathrm{AF} 8$ & 140 & 4 & 0 \\
\hline $\mathbf{1 4 0 0}$ & $\# 397 \mathrm{EFF}$ & 145 & 4 & 0 \\
\hline
\end{tabular}

Table 2. Values of the parameters used in the realization of the Chroma key effect for the front light of 200 lux

\begin{tabular}{ccccc}
\hline $\begin{array}{c}\text { Back } \\
\text { light } \\
{[\mathbf{l u x}]}\end{array}$ & $\begin{array}{c}\text { Color } \\
\text { Code }\end{array}$ & $\begin{array}{c}\text { Color } \\
\text { Toler- } \\
\text { ance }\end{array}$ & $\begin{array}{c}\text { Edge } \\
\text { Thin }\end{array}$ & $\begin{array}{c}\text { Edge } \\
\text { Feather }\end{array}$ \\
\hline $\mathbf{2 0 0}$ & $\# 121526$ & 32 & 3 & 17 \\
\hline $\mathbf{4 0 0}$ & $\# 172041$ & 37 & 3 & 10.5 \\
\hline $\mathbf{6 0 0}$ & $\# 222 \mathrm{D} 5 \mathrm{C}$ & 41 & 3 & 7 \\
\hline $\mathbf{8 0 0}$ & $\# 202 \mathrm{C} 5 \mathrm{E}$ & 50 & 3 & 5 \\
\hline $\mathbf{1 0 0 0}$ & $\# 2 \mathrm{~A} 3877$ & 68 & 4 & 3.5 \\
\hline $\mathbf{1 2 0 0}$ & $\# 2 \mathrm{C} 4393$ & 91 & 4 & 3 \\
\hline $\mathbf{1 4 0 0}$ & $\# 262 \mathrm{~A} 4 \mathrm{D}$ & 96 & 4 & 0 \\
\hline
\end{tabular}

Table 3. Values of the parameters used in the realization of the Chroma key effect for the front light of 800 lux 


\begin{tabular}{ccccc}
\hline $\begin{array}{c}\text { Back } \\
\text { light } \\
{[\mathbf{l u x}]}\end{array}$ & $\begin{array}{c}\text { Color } \\
\text { Code }\end{array}$ & $\begin{array}{c}\text { Color } \\
\text { Toler- } \\
\text { ance }\end{array}$ & $\begin{array}{c}\text { Edge } \\
\text { Thin }\end{array}$ & $\begin{array}{c}\text { Edge } \\
\text { Feather }\end{array}$ \\
\hline $\mathbf{2 0 0}$ & $\# 242 \mathrm{~A} 4 \mathrm{~B}$ & 40 & 1 & 18 \\
\hline $\mathbf{4 0 0}$ & $\# 25294 \mathrm{C}$ & 43 & 1 & 10 \\
\hline $\mathbf{6 0 0}$ & $\# 25294 \mathrm{C}$ & 45 & 1 & 7 \\
\hline $\mathbf{8 0 0}$ & $\# 2 \mathrm{E} 3359$ & 50 & 2 & 4.5 \\
\hline $\mathbf{1 0 0 0}$ & $\# 333 \mathrm{~A} 70$ & 68 & 3 & 2.5 \\
\hline $\mathbf{1 2 0 0}$ & $\# 2 \mathrm{E} 3978$ & 83 & 3 & 2 \\
\hline $\mathbf{1 4 0 0}$ & $\# 36458 \mathrm{~A}$ & 98 & 3 & 1 \\
\hline
\end{tabular}

Table 4. Values of the parameters used in the realization of the Chroma key effect for the front light of 1400 lux

From the results obtained, it is noted that the Color Tolerance parameter value increases as the background color becomes brighter. It is seen that the transparency of the color is higher at brighter backgrounds, or smaller in darker backgrounds. Consequently, a brighter rather than darker color is preferable for the realization of the Chroma-effect. One of the solutions would be to increase the brightness of light in the darker backgrounds, which would increase the value of transparency, and thus get better quality video material made in a virtual studio.

The value of the Edge Thin parameter drops from brighter to darker backgrounds. For the brighter colors, the width of the border of key area must be increased, that is, the transparent area is enlarged. As for the darker colors it is necessary to decrease width of the border of key area. The Edge Feather parameter grows when the background becomes darker. On the basis of the obtained values it can be concluded that the darker colors rather than brighter ones should form more soft edges.

In Fig. 9, a comparison of the Color Tolerance with respect to the intensity of the back light is shown.

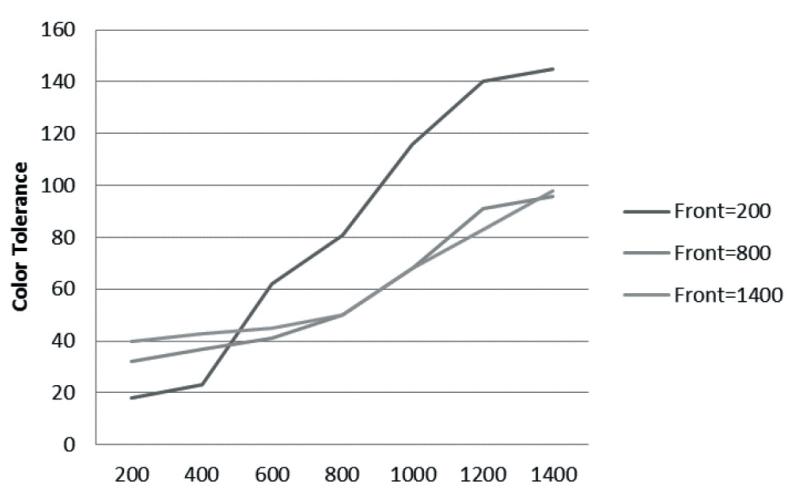

Fig. 9. Changes in Color Tolerance depending on the intensity of the back light.
From Fig. 9 it can be seen that in the front light at a constant value of 200 lux, by increasing the back light after 500 lux, the Color Tolerance value has much more value compared to the front light, while before it had a significantly lower value. When the front light has a constant value of 800 and 1400 lux, the increase in the back light increases Color Tolerance, but with similar mutual changes in value. It can also be concluded that with the change of the backlight up to 800 lux, the Color Tolerance changes are less, and then the values drastically jump.

\section{CONCLUSION}

Based on the results obtained by analyzing the background in the Chroma key effect in a virtual studio for different illumination of the blue background, it can be concluded that the lighter shades of blue color are better for the realization of the Chroma key effect and are therefore more favorable for the background. It also concludes that the change in illumination significantly influences the quality of the Chroma key effect. Degradation of quality is very noticeable at the lowest values of light, both the back and the front (more pronounced at the front). Already at 800 and 1400 lux lighting values, we get a correct Chroma key effect, where the lower values of the last illumination of 200-400 lux degrade the quality, after which the effect is at a satisfactory level. The most optimal results were obtained for values of front light of 800 and 1400 lux, with a back light ranging from 600 to 1200 lux.

The virtual studio compared to conventional production has many advantages such as cost savings on material for scenery, simply changing the entire scenery. Studio is used more efficiently, simply changing the shape and texture of computer-generated scenery, a small studio visually looks much larger. The experience of numerous broadcasters and production companies shows that the current technology of virtual studio is equally suitable for all types of television programs: news, weather, sports shows, advertisements, quizzes, choices, music and children's educational programs, real-time programs with audiences as well as short television forms.

\section{ACKNOWLEDGMENT}

This work was done within the project Implementation of the study program - Digital Broadcasting and Broadband Technologies (DBBT Master studies), Erasmus+ CBHE Project No. 561688-EPP-1-2015-1-XKEPPKA2-CBHE-JP. 


\section{REFERENCES}

[1] M. Moshkowitz, The Virtual Studio: Technology and Techniques, Focal Press: Waltham, 2000.

[2] M. Petrovic, Televizija, Faculty of Technical Sciences: Kosovska Mitrovica, 2007.

[3] S. Wright, Digital Compositing for Film and Video, Focal Press: Waltham, 2010.

[4] N. T. Sang and T. Q.Vinh, "FPGA Implementation for Real-time Chroma-key Effect Using Coarse and Fine Filter," in Proc. of International Conference on Computing, Management and Telecommunications (ComManTel), Ho Chi Minh City, Vietnam, January 21-23, 2013, pp. 157-162.

[5] B. Vidal, "Chroma Key Visual Feedback Based on Non-Retroreflective Polarized Reflection in Retroreflective Screens," IEEE Transactions On Broadcasting, vol. 58, no. 1, pp. 144-150, March 2012.

[6] O. Grau, T. Pullen and G. A. Thomas, “A combined studio production system for 3-d capturing of live action and immersive actor feedback," IEEE Transactions on Circuits and Systems for Video Technology, vol. 14, no. 3, pp. 370-380, March 2004.

[7] B. Vidal, "Chroma key visual feedback based on non-retroreflective polarized reflection in retroreflective screens," IEEE Trans. Broadcast., vol. 58, no. 1, pp. 144-150, Mar. 2012.

[8] B. Jakšić, B. Gara, M. Petrovic, P. Spalevic and Lj. Lazic, "Analysis of the Impact of Front and Back light on Image Compression with SPIHT Method during Realization of the Chroma Key Effect in Virtual TV Studio," Acta Polytechnica Hungarica, vol. 12, no. 2, pp. 71-88, 2015.

[9] R. Brinkmann, The Art and Science of Digital Compositing, Second Edition: Techniques for Visual Effects, Animation and Motion Graphics. Morgan Kaufmann: Burlington, 2008.
[10] C. R. Dyer and L. S. Davis, Volumetric scene reconstruction from multiple views, Foundations of Image Understanding, Kluwer: Boston, 2001, pp. 469-489.

[11] M. Petrovic and B. Jaksic, Analysis of Lighting Impact in the Realization of Virtual TV Studio, LAP Lambert Academic Publishing: Saarbrücken, 2015.

[12] B. Fitt and J. Thornley, Lighting Technology: A Guide for the Eentertainment Industry, 2nd ed, Elsevier: Amsterdam, 2001.

[13] N. Mobsby, Lighting Systems for TV Studios, Entertainment Technology Press Ltd: Cambridge, 2001.

[14] Minolta Color Meter - technical specifications: http://www.cameramanuals.org/minolta_pdf/minolta_color_meter_ii.pdf

[15] Digital Light Meter - technical specifications: http:// www.electronickits.com/gold/lt2101.htm.

[16] Canon EOS 550D - technical specifications: https:// www.canon.co.uk/for_home/product_finder/cameras/digital_slr/eos_550d/.

[17] U. Fecker; M. Barkowsky and A. Kaup, "HistogramBased Prefiltering for Luminance and Chrominance Compensation of Multiview Video," IEEE Transactions on Circuits and Systems for Video Technology, vol. 18, no. 9, pp. 1258-1267, 2008.

[18] T. Jiang. K. D. Kuhnert, D. Nguyen and Lars Kuhnert, "Multiple templates auto exposure control based on luminance histogram for onboard camera," in Proc. of IEEE International Conference on Computer Science and Automation Engineering (CSAE), Shanghai, China, June 10-12, 2011, vol. 3, pp. 237-241.

[19] P. Lin , C. Lin and H. Yen, “Tri-histogram equalization based on first order statistics, " in Proc. of 13th IEEE International Symposium on Consumer Electronics, Kyoto, Japan, May 25-28, 2009; pp. 387-391. 